\title{
Actividades Complementarias - Extraescolares y el aprendizaje en Estudiantes de Básica Primaria: un Estudio con Análisis en Correspondencias Múltiples
}

\section{Impact of Supplementary and Extracurricular Activities to Students Learning of Primary Basic: a Study with Multiple Correspondence Analysis}

DOI: $10.54018 /$ seesv3n1-013

Recebimento dos originais: 01/01/2021 Aceitação para publicação: 01/02/2022

\section{Daniel Enrique Támara López}

Magister en Ciencias - Matemática Aplicada, Universidad Nacional de ColombiaSede Manizales.

Universidad de Sucre- Grupo de investigación Estadística y Modelamiento Matemático Aplicado a Calidad Educativa.

Cra 28 \# 5-267 Barrio Puerta Roja - Sincelejo (Sucre). Bloque 5-Salon 106.

E-mail: detamaral@unal.edu.co

\section{Estefanía Julieth Guevara Blanquicett}

Magister en Ciencias - Matemática Aplicada, Universidad Nacional de ColombiaSede Manizales.

Universidad de Sucre- Grupo de investigación Estadística y Modelamiento Matemático Aplicado a Calidad Educativa.

Cra 28 \# 5-267 Barrio Puerta Roja - Sincelejo (Sucre). Bloque 5-Salon 106. E-mail: ejguevarab@unal.edu.co

\section{María Clareth Méndez Ramos}

Especialista en Estadística Aplicada, Fundación Universitaria Los Libertadores. Universidad de Sucre- Grupo de investigación Estadística y Modelamiento Matemático Aplicado a Calidad Educativa.

Cra 28 \# 5-267 Barrio Puerta Roja - Sincelejo (Sucre). Bloque 5-Salon 106. E-mail: mariaclare29@gmail.com

\section{Andrea Carolina Menco Tovar}

Licenciada en Matemáticas, Universidad de Sucre Universidad de Sucre- Grupo de investigación Estadística y Modelamiento Matemático Aplicado a Calidad Educativa.

Cra 28 \# 5-267 Barrio Puerta Roja - Sincelejo (Sucre). Bloque 5-Salon 106. E-mail: andreacarolinamenco1996@gmail.com

\section{Melba Liliana Vertel Morinson}

Magister en Ciencias Estadísticas, Universidad Nacional de Colombia. Docente titular. Universidad de Sucre- Líder del Grupo de investigación Estadística y Modelamiento Matemático Aplicado a Calidad Educativa. Cra 28 \# 5-267 Barrio Puerta Roja - Sincelejo (Sucre). Bloque 5-Salon 106. E-mail: melba.vertel@unisucre.edu.co 


\section{RESUMEN}

El principal objetivo del trabajo fue caracterizar la posible incidencia en la calidad educativa de estudiantes de básica primaria del municipio de Sincelejo (SucreColombia), de la realización de actividades extraescolares y complementarias, según su tipología (recreativas, cognitivas o ambas). Se utilizó un muestreo por conglomerados (escuelas) y estratificado en dos etapas (administradores y estudiantes de la escuela), administrando a estudiantes pruebas estandarizadas de rendimiento académico (resultados pruebas saber $3^{\circ}, 5^{\circ}$ y $9^{\circ}$ ) suministrada por el ICFES (2012) y un instrumento diseñado ad-hoc (encuesta) para existencia y características de actividades complementarias y extraescolares en la escuela. Analizaremos la relación existente entre variables similares, mediante la metodología estadística Análisis de Correspondencias Múltiples (ACM). Para desarrollar el análisis estadístico se usó el lenguaje estadístico $\mathrm{R}$, los paquetes ade4 y FactoClass. Los resultados de los perfiles muestran que la realización de algún tipo de actividad complementaria o extraescolar, sea del tipo que sea, mejora el rendimiento académico de los estudiantes de básica primaria. También, muestra que se favorece el desarrollo de capacidades afectivas, motoras y cognitivas en la población infantil que tienen mayor acceso a este tipo de actividades.

Palabras clave: Actividades Complementarias, Actividades Extraescolares, Calidad Educativa, Análisis En Correspondencias Múltiples, Lenguaje Estadístico R.

\section{ABSTRACT}

The main objective of the study is to estimate the impact that brings the implementation of extracurricular and complementary activities to the quality education of students in basic primary municipality of Sincelejo. It is based on information obtained through surveys, official institutions and private and from data supplied by ICFES (results tests know $3^{\circ}, 5^{\circ}$ and $9^{\circ}, 2012$ ). Likewise, compare the found finds and are subject to study in multiple correspondence analysis, technique which produced planes factorial crossing the level of education of the schools, based on the evidence to know, with the existence and characteristics of extracurricular and complementary activities. To develop the statistical analysis the statistical language $R$, packets ade4 and FactoClass was used. He is achieved conclude that avoids most influence these activities into non-formal schools, generally satisfactory and advanced levels are accompanied in a high percentage with them.

Keywords: Complementary Activities, Extracurricular Activities, Educational Quality, Multiple Correspondence Analysis, Statistical Language R.

\section{INTRODUCCIÓN}

En la actualidad, el rendimiento académico es un tema que sigue suscitando un enorme interés, no solo a nivel social (Raimundi et al, 2014) y académico (Escudero, 2005) sino también a nivel de investigación (Cladellas et 
al, 2013). Es por lo anterior, que se han llevado a cabo numerosos estudios al respecto tratando de identificar cuáles son los factores de mayor contribución, con lo que se ha llegado a demostrar que la familia (Bolívar 2006, Cervini et al 2014), la motivación y la autoestima (Navas et al 2003, Schmidt y Padilla 2003, Clariana et al 2014), los medios de comunicación, la cultura y en general la sociedad (Pereira 2003) en la cual viven los escolares son los entes de mayor influencia.

A nivel conductual algunos estudios (sobre todo en la década del 20002010) han investigado la relación entre actividades complementariasextraescolares y rendimiento académico. También, posibles beneficios y consecuencias que estas aportan a la calidad educativa de los estudiantes. El propósito en esta investigación fue investigar la realidad que viven las instituciones educativas del municipio de Sincelejo (Sucre - Colombia) en lo que se refiere al esfuerzo que hacen para complementar la formación de los estudiantes mediante la preparación y ejecución de actividades complementarias-extraescolares y, del mismo modo, deducir cuál es el impacto que trae la implementación de ellas a la calidad educativa. Ya que una de las metas más importantes en cualquier política educacional es resolver el problema de la desigualdad educacional en relación al estrato socioeconómico de los estudiantes y las escuelas.

Inicialmente se describirán características de lo realizado a una muestra de 25 escuelas ( 11 oficiales y 14 privadas), concernientes a escenarios académicos (salas de sistema, de audiovisuales y laboratorios) y deportivos, acompañamiento a estudiantes, condiciones y uso de bibliotecas, todo esto enmarcado dentro de lo que se conoce como actividades complementarias. De igual forma, se detallan aquellas actividades extraescolares (desarrollo artístico y deporte) que permiten potenciar en el estudiante la capacidad de relacionarse con su medio social y expandir su horizonte cultural.

Posteriormente, como el estudio se centra en los procesos internos de las escuelas, y para ello se usó métodos de investigación cualitativa se realizó el análisis estadístico, basado en el método de análisis en correspondencias múltiples, técnica con la que se logró reducir la variabilidad del conjunto de individuos (escuelas) para identificar con mayor precisión y facilidad aquellas de mayor influencia en el estudio.

Es de importancia notar que en el desarrollo del trabajo se hace continuamente un paralelismo entre escuelas oficiales y no oficiales, y la razón de 
esto es la de visualizar en ellas la distancia que hay entre sus condiciones tecnológico-logísticas y el modo en que estructuran los procesos de formación.

\section{DESCRIPCIÓN DE ACTIVIDADES COMPLEMENTARIAS}

Por Actividades Complementarias ha de entenderse aquellas realizadas con fines netamente instructivos y por lo general ocurridas en horarios académicos. Como el nombre lo indica buscan complementar la formación del educando mediante la ejecución de planes formativos (implementación de un servicio de refuerzo estudiantil, opciones de nivelación y recuperación, entre otros) y uso de herramientas y espacios didácticos (bibliotecas, salas virtuales, científicas y deportivas). A continuación, se describen las actividades complementarias, divididas en tres categorías: Escenarios Académicos y Deportivos, Acompañamiento a Estudiantes y Uso y Condiciones de Bibliotecas, que desarrollan las instituciones con nivel de básica primaria en el municipio de Sincelejo.

\subsection{ESCENARIOS ACADÉMICOS Y DEPORTIVOS}

El uso de la tecnología en la escuela no solo favorece los procesos de enseñanza y aprendizaje, un efecto mayor se verifica en la motivación que supone haber en los estudiantes debido al dinamismo e interactividad que se consigue con la manipulación de la misma; al respecto, en Barragán y Ruiz (2001) se expresó que incluir material didáctico en el ambiente escolar contribuye a fomentar el desarrollo de múltiples aprendizajes, especialmente en niños y niñas en edades tempranas, en los cuales implementar estas estrategias promueve el desarrollo lógico-matemático, lingüístico, entre otras habilidades psicomotrices y culturales.

Por otro lado, la actividad física se considera hoy día como acción fundamental para el óptimo desarrollo del ser humano, sobre todo si es practicada desde edades tempranas pues favorece al desarrollo de capacidades afectivas, motoras y cognitivas. En Mijangos (2005), se expresa que una de la razón fuerte por las cuales se presenta el fracaso escolar es debido a la falta de incorporación de la dimensión corporal y la utilización de aprendizajes escolares clásicos y rígidos, en otras palabras, afirma en este se afirma que existe una relación directa entre el desarrollo motriz y el rendimiento escolar. 
En las siguientes líneas se describen varios aspectos sobre las instalaciones virtuales, científicas y deportivas que ofrecen las escuelas con niveles de básica primaria en el municipio de Sincelejo. De 21 escuelas, públicas y privadas, que tienen salas de sistema solo 6 cuentan con recursos suficientes, no todos los estudiantes pueden usar a la vez, por ejemplo, computadoras en clase de tecnología. Además, se hace necesario en algunos casos elaborar convenios con entidades externas para llegar a usar tales herramientas. Para el caso de salas de audiovisuales, 9 de 25 colegios, es decir, el 36\%, tienen este espacio con recursos suficientes.

Tabla 1: Instalaciones virtuales - Salas de sistemas

\begin{tabular}{|l|c|c|c|c|c|}
\hline \multicolumn{7}{|c|}{ Tienen } & $\%$ & $\begin{array}{c}\text { Salas de Sistema } \\
\text { Recursos } \\
\text { necesarios }\end{array}$ & $\begin{array}{c}\text { Recursos } \\
\text { suficientes }\end{array}$ & Convenios \\
\hline Públicas & 8 & 72.7 & 8 & 1 & 1 \\
\hline Privadas & 13 & 92 & 13 & 5 & 1 \\
\hline
\end{tabular}

Fuente: Elaboración propia

Tabla 2: Instalaciones virtuales - Salas de audiovisuales

\begin{tabular}{|l|c|c|c|c|c|}
\hline \multicolumn{7}{|c|}{ Tienen } & $\%$ & $\begin{array}{c}\text { Recursos } \\
\text { necesarios }\end{array}$ & $\begin{array}{c}\text { Recursos } \\
\text { suficientes }\end{array}$ & Convenios \\
\hline Públicas & 4 & 36.4 & 4 & 4 & 1 \\
\hline Privadas & 12 & 85.7 & 12 & 5 & 1 \\
\hline
\end{tabular}

Fuente: Elaboración propia

En lo concerniente a instalaciones científicas se encontró que ninguna escuela ofrece tal servicio para niveles de básica primaria, lo hacen exclusivamente para grados superiores.

Finalmente, en cuanto a instalaciones deportivas se resalta que ningún colegio con solo niveles de básica primaria tiene verdaderos espacios deportivos, la actividad física se desarrolla en algunos casos fuera del centro educativo.

\subsection{ACOMPAÑAMIENTO A ESTUDIANTES}

Un servicio de refuerzo estudiantil (S.R.E.) es un sistema de atención al estudiante que busca reforzar la actividad académica y hacer seguimiento cercano de los procesos de aprendizaje. Puede decirse que la intención principal de un S.R.E. es la de identificar problemas académicos en los escolares y tratar de disminuirlos mediante un acompañamiento continuo y flexible. A nivel internacional se han implementado programas de acompañamiento estudiantil en 
escuelas de educación básica consiguiendo resultados positivos en distintas dimensiones. Según (Rivière, 2008) estos programas de acompañamiento escolar permiten a los centros escolares mejorar sus indicadores tanto en el ámbito del rendimiento académico como en términos de la convivencia y la armonía entre el alumnado. Así mismo los estudiantes que participan de estos programas cambian su dinámica de forma positivas, recuperando la motivación y mejorando su comportamiento en clases. A continuación, se describen características del S.R.E. que implementan algunas escuelas de educación primaria.

Existen escuelas oficiales y no oficiales que no cuentan dentro de su plan de estudios con un servicio de refuerzo estudiantil. Las razones son variadas, entre ellas, la no disponibilidad del personal docente (pues en algunos casos laboran en jornada contraria) para apoyar y reforzar a los estudiantes, la mala interpretación de la estrategia: padres de familia exigían la aprobación de las materias de sus hijos por el solo hecho de estos asistir a refuerzos y tutorías, entre otras.

Es preciso señalar que en algunos colegios no oficiales la realización de S.R.E. es obligatorio pues está incluido dentro del proyecto educativo institucional y es tarea de los docentes diseñar, ejecutar y evaluar la acción de acompañamiento realizada. En este sentido, aunque no exista la necesidad académica el ejercicio debe llevarse a cabo y usar el espacio para adelantar temáticas, preparar actos culturales y más. En la tabla 3 se resume la existencia y algunas características de los Servicios de Refuerzo Estudiantil de las escuelas con niveles de básica primaria.

Tabla 3: Servicio de Refuerzo Estudiantil En Colegios Con Básica Primaria.

\begin{tabular}{|r|c|c|c|c|}
\hline \multicolumn{6}{|c|}{ Servicio de Refuerzo Estudiantil } & \multicolumn{4}{|c|}{ Personal a } & Lugar \\
\hline & Tiene & $\%$ & cargo & Colegio \\
\hline Públicas & 7 & 63.6 & $\begin{array}{c}\text { Practicantes } \\
\text { y Docentes }\end{array}$ & Colegio \\
\hline Privadas & 8 & 57.1 & Docentes &
\end{tabular}

Fuente: Elaboración propia

\subsection{CONDICIONES Y USO DE BIBLIOTECAS}

Un lugar en el cual afianzar y expandir conceptos, consultar bibliografía y desarrollar hábitos de lectura debería existir en toda institución educativa, es por ello que según (Peacock, 1961), el impacto más importante que se espera obtener 
en los niños al terminar su camino por la escuela es la motivación y el hábito de la lectura académica y recreativa, para lo cual los niños deben contar primordialmente en su contexto educativo con libros que incentiven el interés por esta y que además promueva la inteligencia y sensibilidad.

La tabla 4 indica el porcentaje de escuelas públicas y privadas de primaria del municipio de Sincelejo que cuentan con el recurso de biblioteca.

Tabla 4: Bibliotecas de las Escuelas con Básica Primaria
\begin{tabular}{|l|l|l|}
\hline \multicolumn{3}{|c|}{ Bibliotecas } \\
\hline Pública & 6 & $\%$ \\
Privada & 11 & 54.5 \\
\end{tabular}

Fuente: Elaboración propia

La escases de recursos bibliográficos y de espacios para la adecuación de los mismos son la principal dificultad a la que se enfrentan las escuelas de educación primaria. En cuanto al uso que dan los alumnos a los implementos propios de ellas, como libros, computadores y demás, afirman docentes que está desviado y encaminado a fines de entretenimiento, expresan además que la formación de un hábito de lectura en tiempos como los actuales es muy difícil de consolidar en los niños dada la forma en que se maneja hoy día la tecnología y el impacto que tienen las corrientes facilistas en la actitud y rendimiento académico de los mismos.

\section{DESCRIPCIÓN DE ACTIVIDADES EXTRAESCOLARES}

Entiéndase por Actividades Extraescolares aquellas realizadas en horarios no necesariamente académicos con intención de generar competencias personales y ciudadanas. Puede decirse que el principal propósito de tales actividades es contribuir al bienestar integral de los estudiantes por medio de actividades de diversa índole que van desde las deportivas, culturales, tecnológicas cognitivas, etc. (Álvarez et al.).

A continuación, se describen las actividades extraescolares que llevan a cabo las escuelas con niveles de básica primaria. 


\subsection{DESARROLLO ARTÍSTICO}

La expresión "Desarrollo Artístico" agrupa un conjunto de actividades que buscan principalmente el fortalecimiento de destrezas personales, tales como la habilidad para la danza, la pintura, las manualidades, etc. En esta línea, hay estudios que revelan que la influencia de las manifestaciones artísticas presentes en el entorno causa gran impacto en el desarrollo de algunas capacidades físicas y mentales en niños de seis a doce años.

En (Cantero,2011) se expresa que la educación artística es un área que incentiva la visión y la expresión de los estudiantes desde el punto de vista artístico pero que a su vez esta puede servir de herramienta facilitadora de aprendizaje en los mismo desde cualquier área en general o especifica.

Seguidamente se mostrarán y describirán las actividades artísticas que ofrecen los colegios para los niños en ciclos de básica primaria.

Tabla 5: Desarrollo Artístico En Las Instituciones Con Básica Primaria.

\begin{tabular}{|r|c|l|l|l|}
\hline \multicolumn{4}{|c|}{ Desarrollo Artístico } \\
\hline & Danza & Pintura & Manualidades & Otro \\
\hline Públicas & 5 & 2 & 0 & 0 \\
\hline Privadas & 6 & 5 & 4 & 2 \\
\hline
\end{tabular}

Fuente: Elaboración propia.

Sobre Desarrollo Artístico: En ocho (8) escuelas no oficiales, la creación de espacios para el desarrollo de destrezas artísticas tiene importancia dentro de la planificación general del proyecto institucional, existen horarios establecidos, personal exclusivo y se dedica tiempo y recursos para llevar a cabo tales actividades. Para el resto de instituciones no oficiales y para todas las públicas la situación cambia considerablemente, la forma en que se dan estas prácticas es irregular, clases de canto, por ejemplo, son dadas eventualmente en algunas asignaturas como metodología para desarrollar un tema.

\subsection{DEPORTES}

Muchos autores dan su opinión acerca de la importancia del deporte educativo, por ejemplo, en Corrales (2010), se expresa que es más que una práctica física o medio de canalización del tiempo libre, este se constituye como un espacio educativo con énfasis e impacto social.

Desde este punto de vista el desarrollo de eventos deportivos en centros 
educativos es justificable y por ello a continuación se describen los actos deportivos que son desarrollados conjuntamente por las escuelas de básica primaria en el municipio de Sincelejo.

Sobre Eventos Deportivos, por lo general el desarrollo de eventos deportivos se da dentro de jornadas culturales, que son actos a los que asisten gran cantidad de colegios, oficiales y no oficiales, para exponer proyectos científicos, participar en olimpiadas y ejecutar otro tipo de actividades. Fuera de las jornadas culturales la acción deportiva se limita a la clase de educación física.

\section{ANÁLISIS ESTADÍSTICO: ANÁLISIS EN CORRESPONDENCIAS MÚLTIPLES}

El análisis de correspondencias múltiples (ACM) se utiliza para estudiar tablas de individuos (colegios) con variables cualitativas o categóricas. Es adecuado para analizar las asociaciones entre variables de individuos, abordar el análisis multivariado de encuestas y para explotar bases de datos con información cualitativa (Pardo y Cabarcas, 2001).

Para desarrollar el análisis estadístico se usó el lenguaje estadístico $R(R$ DevelopmentCoreTeam, 2011), los paquetes ade4 (Thioulouse et al., 2004) y FactoClass (Pardo y Del Campo, 2007).

La información obtenida fue dividida en grupos (ellos contienen parte de las variables consideradas), cada uno estudiado inicialmente de manera individual mediante el método análisis en correspondencias múltiples (ACM). Se destacó de cada grupo cuáles eran los individuos y variables que mejor calidad de representación tenían y así mismo, de sus respectivos planos factoriales se indicó el porcentaje con que se representan sus variables de inicio. Al final se analiza de manera conjunta en un plano factorial todas las variables consideradas haciendo un estudio similar al que se hizo para cada grupo.

A continuación, se presenta el análisis hecho a cada grupo de variables. 
Fig.1: Plano Factorial Salas de Sistema (Existencia, Condiciones e Intensidad Horaria Semanal)

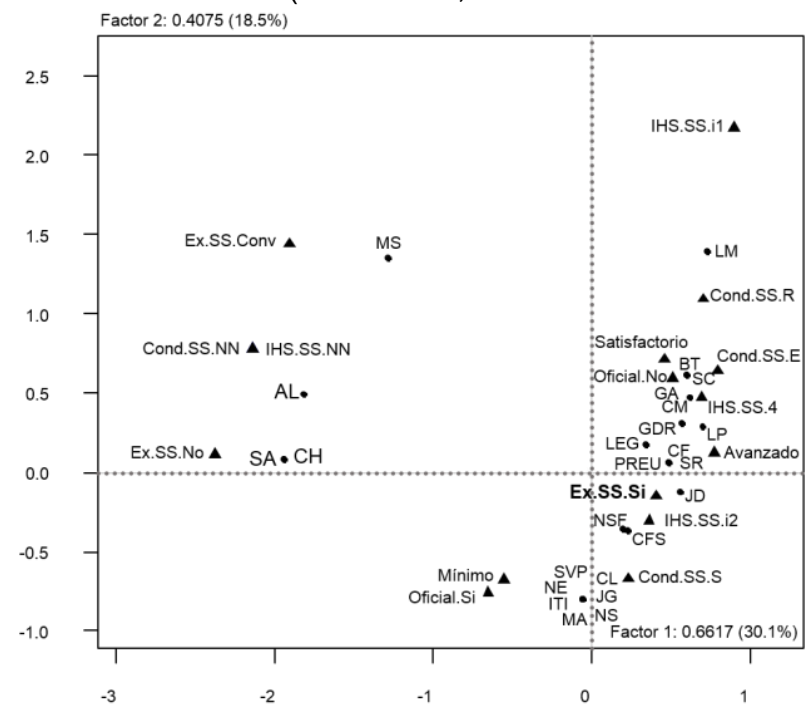

Fuente: Elaboración propia.

La inercia presentada en este plano factorial es de 1.0692, dividida entre los dos primeros valores propios en un $48.6 \%$ (Fig. 1). Para este caso las variables originales están representadas en un $48.6 \%$. La variable de mayor contribución es la existencia de las salas de sistemas (EX.SS.SI), solo el 16\% de las instituciones encuestadas, ubicadas en el segundo cuadrante del plano factorial, no cuentan con estas aulas. Se nota una gran acumulación de puntos entre las escuelas con nivel satisfactorio o avanzado que poseen salas de sistema.

Fig.2: Plano Factorial Salas de Sistema (Recursos Necesarios y Suficientes).

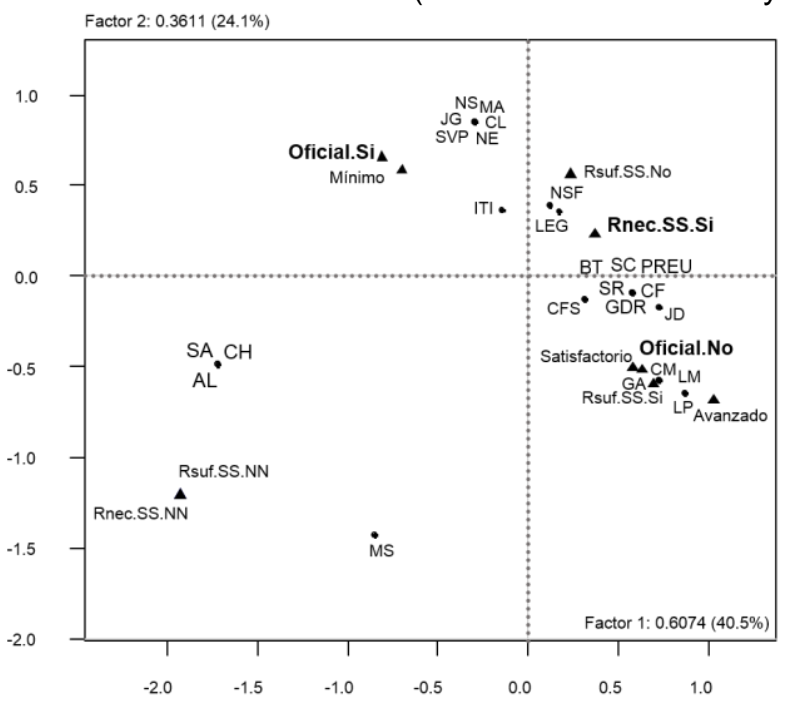

Fuente: Elaboración propia. 
Las variables iniciales están representadas en un $64.6 \%$ por las nuevas componentes, mismo porcentaje en el cual se acumula la inercia entre los dos primeros valores propios (Fig. 2). Evidentemente sobresale la existencia de recursos necesarios para las salas de sistema, pero del $84 \%$ de instituciones que cuentan con tales implementos, solo el $24 \%$ tiene recursos suficientes, para estos últimos, a excepción de ITI, los niveles presentados son satisfactorio o avanzado.

Se muestran a continuación los planos factoriales de las salas de audiovisuales junto con su existencia, condiciones, intensidad horaria semanal y recursos necesarios y suficientes.

Fig.3: Plano Factorial Salas de Audiovisuales (Existencia, Condiciones e Intensidad Horaria Semanal)

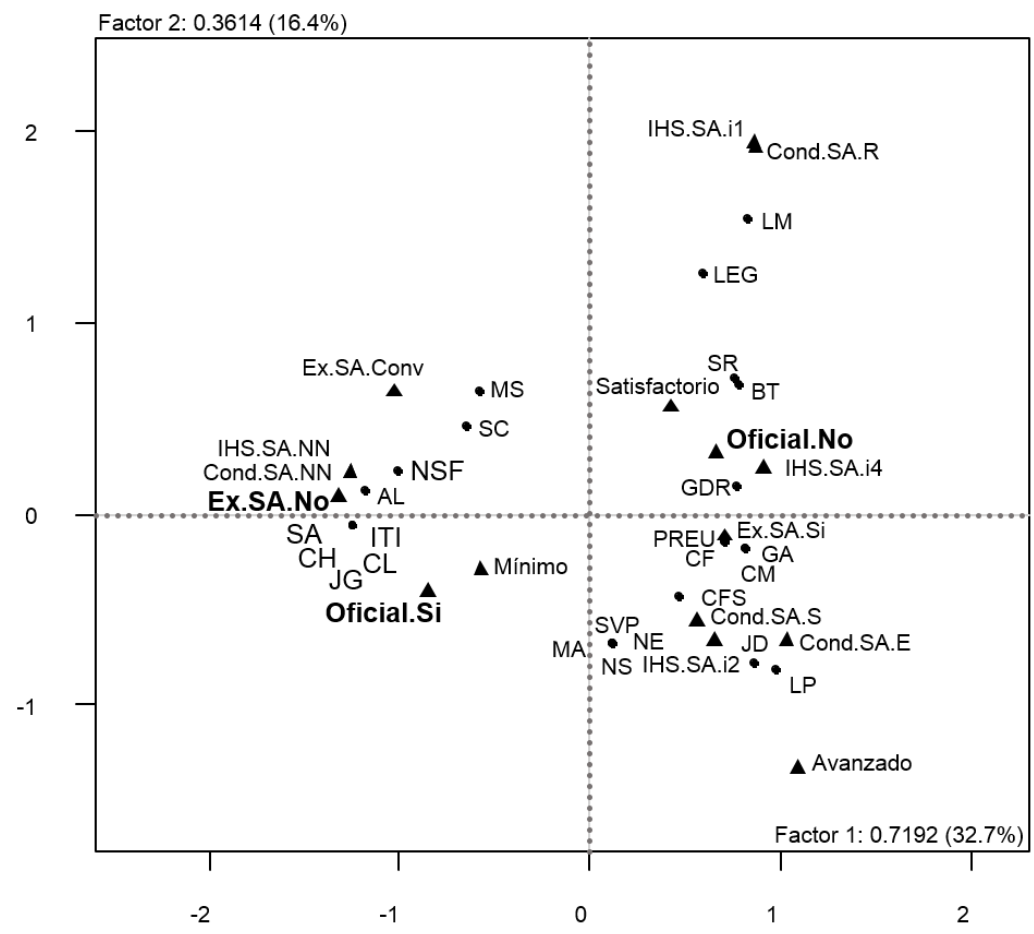

Fuente: Elaboración propia.

En este plano factorial la inercia total es de 1.0806, acumulada en un $49.1 \%$ entre los dos primeros valores propios, es decir, el plano factorial representa en un $49.1 \%$ las variables iniciales(Fig. 3). Se tiene como caso particular que instituciones no oficiales con salas de audiovisuales propias en condiciones favorables mantienen en su gran mayoría un nivel satisfactorio. En contraste, del $63.3 \%$ de colegios públicos que no tienen salas de audiovisuales solo el $9.09 \%$ (correspondiente a NSF) está en rango satisfactorio, el $54.21 \%$ restante se localiza en mínimo. 
Fig.4: Plano Factorial Salas de Audiovisuales (Recursos Necesarios y Suficientes)

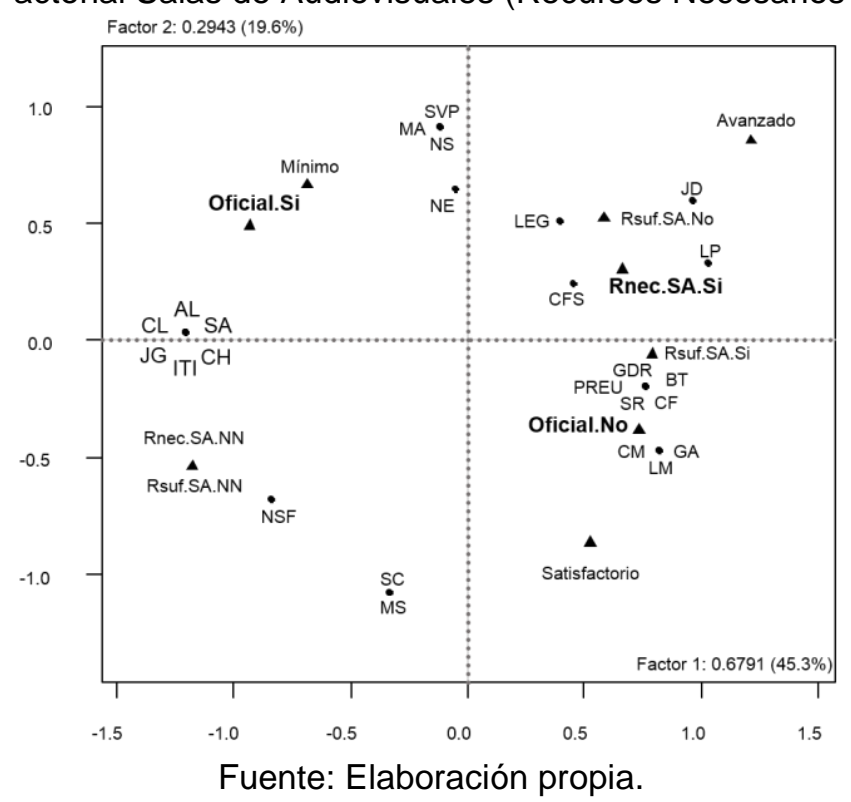

Se tiene una inercia de 0.9734 soportada en los dos primeros valores propios en un $64.9 \%$, o sea el plano factorial representa en este porcentaje las variable iniciales(Fig. 4). Sobresale en el gráfico que colegios no oficiales con salas de audiovisuales dotadas con recursos necesarios y suficientes yacen en nivel satisfactorio y así mismo, que del $63.6 \%$ de escuelas públicas que no tienen tales instalaciones, el $54.5 \%$ está en grado mínimo.

Fig.5: Plano Factorial Acompañamiento a Estudiantes (Existencia e Intensidad Horaria Semanal)

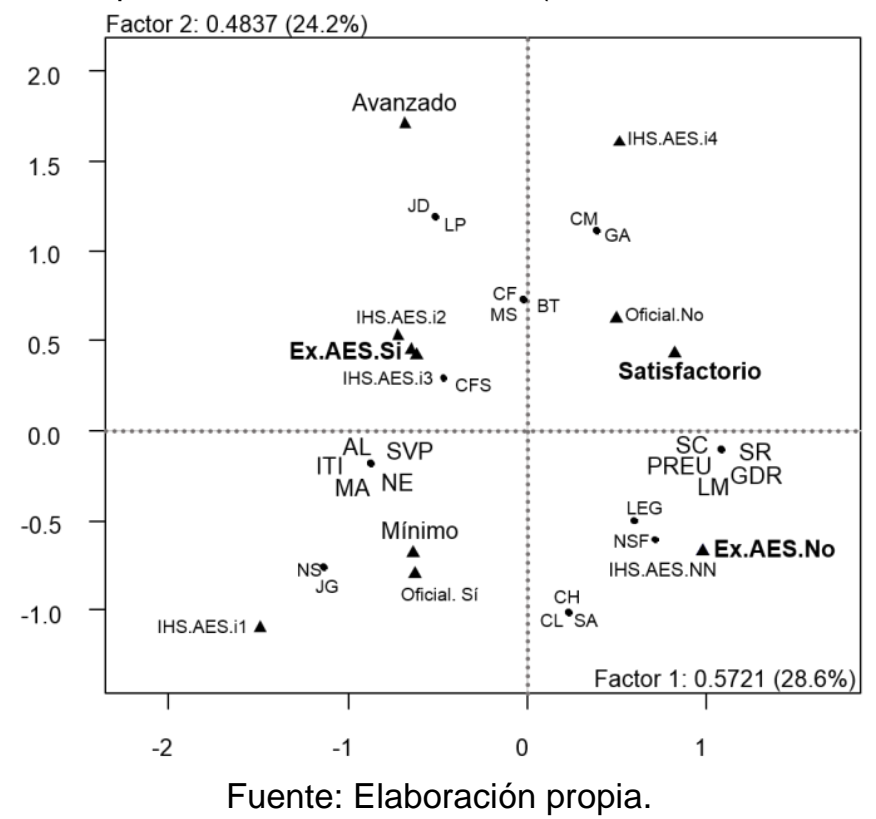


La inercia total asociada del plano factorial es de 1.0558, retenida en un $52.8 \%$ entre los dos primeros valores propios, significando esto que el plano factorial representa en un $52.8 \%$ las variables iniciales(Fig. 5). Se observa que el eje uno opone, por ejemplo, las instituciones ITI, AL, SVP, NE y MA con PREU, SC, SR, GDR y LM, en donde el primer grupo a pesar de contar con S.R.E. reposa en nivel mínimo, a diferencia del segundo que sin tener tal servicio se ubica en nivel satisfactorio. Se detecta también que solo un colegio oficial (NSF) sin sistema de refuerzo se encuentra en grado satisfactorio, el resto, en mínimo. Para el caso de las escuelas no oficiales el $50 \%$ de ellas, correspondiente a 7 instituciones, cuentan con S.R.E. y se hallan entre los grados satisfactorio y avanzado.

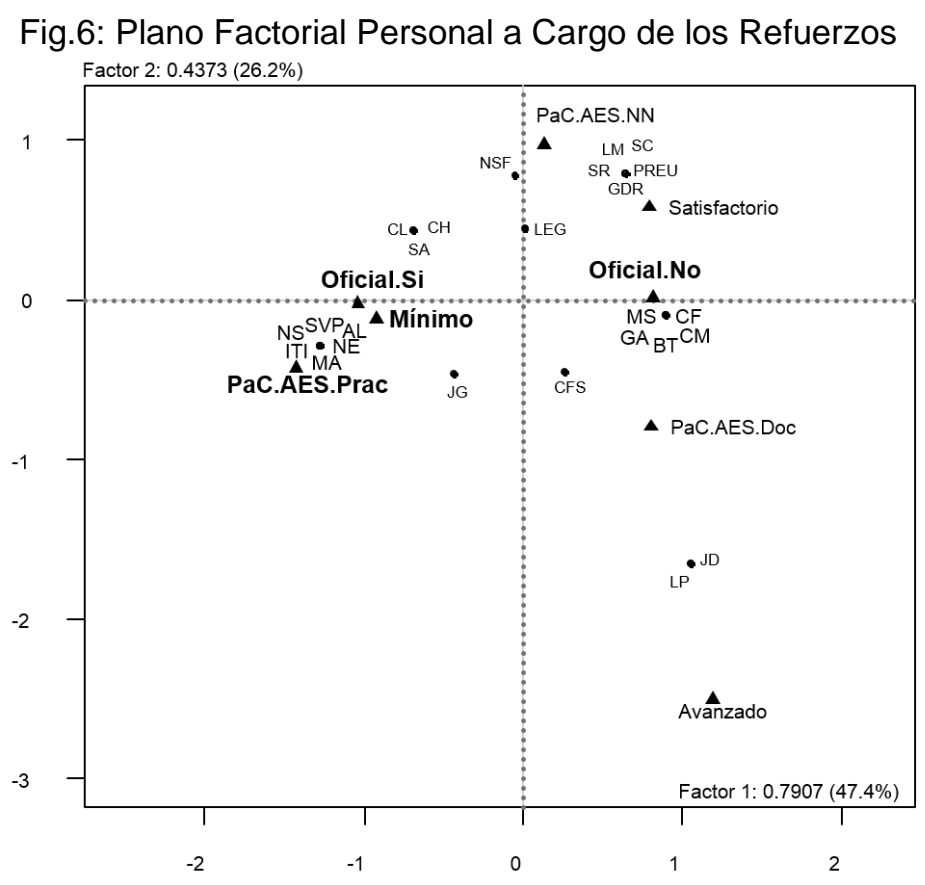

Fuente: Elaboración propia.

$73.6 \%$ es el grado de representatividad que tiene las variables iniciales en este plano factorial. De la inercia total de las variables el 1.228 se encuentra distribuida entre los dos primeros valores propios(Fig. 6). Claramente se nota que las instituciones oficiales que tienen, por lo general, a los practicantes como acompañantes en los servicios de refuerzo para los escolares, están en nivel mínimo.

Ahora se analizarán los planos factoriales que involucran las bibliotecas con su existencia, condiciones, uso dado por estudiantes y docentes. 
Fig.7: Plano factorial Bibliotecas (Existencia y Condiciones)

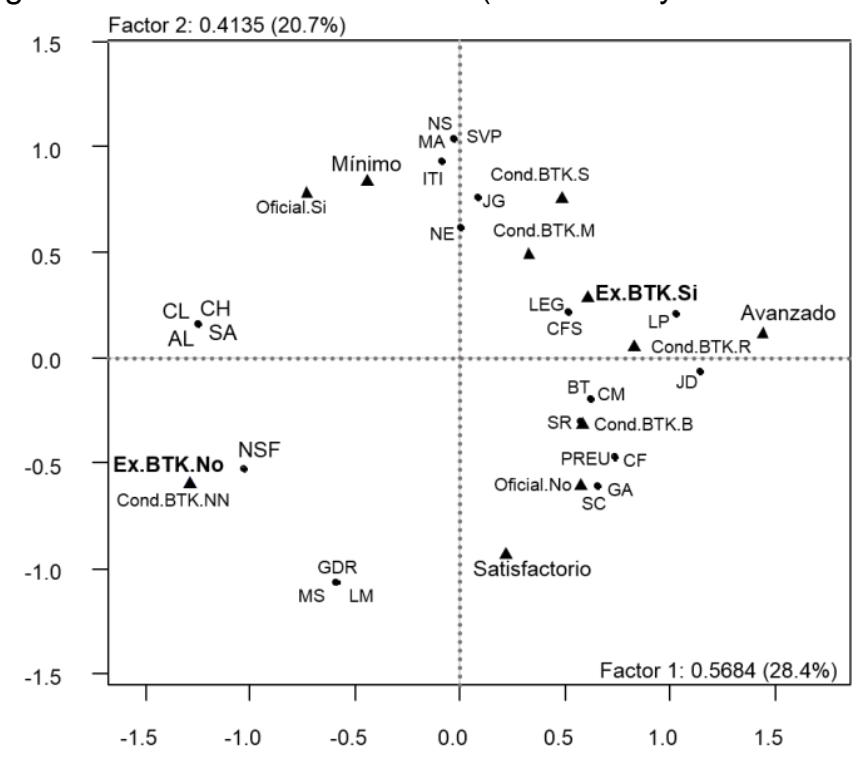

Fuente: Elaboración propia.

La inercia total asociada al plano es de 0.9819, la cual está acumulada en un $49.1 \%$ entre los primeros dos valores propios, es decir, hay una representación del $49.1 \%$ de las variables iniciales(Fig. 7). Se observa que el segundo eje opone, por ejemplo, las instituciones públicas $\mathrm{SA}, \mathrm{AL}, \mathrm{CL}$ y $\mathrm{CH}$ con NSF, las primeras 4 con biblioteca y en nivel mínimo y la otra que sin este espacio alcanza un estrato satisfactorio. Por otra parte, la existencia de bibliotecas en las escuelas privadas se asocia a niveles académicos favorables. 
Fig.8: Plano factorial Biblioteca (Uso Dado por Estudiantes y Docentes)

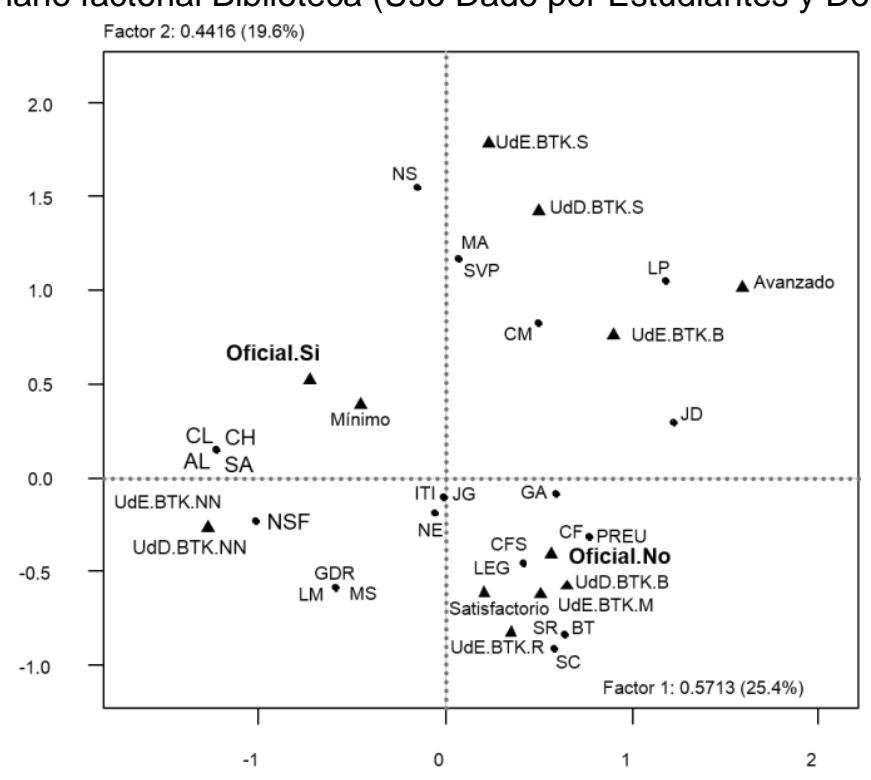

Fuente: Elaboración propia.

La inercia total es de 1.0129, además hay una representación del 45\% de las variables de partida (Fig. 8). El plano factorial revela que son pocas las instituciones en las cuales los estudiantes dan un buen uso a la biblioteca. Las públicas que atienden a tal hecho son aquellas donde las condiciones de la biblioteca son satisfactorias, correspondiendo éstas al $27.3 \%$ del total de ellas.

Fig.9: Plano factorial Desarrollo Artístico

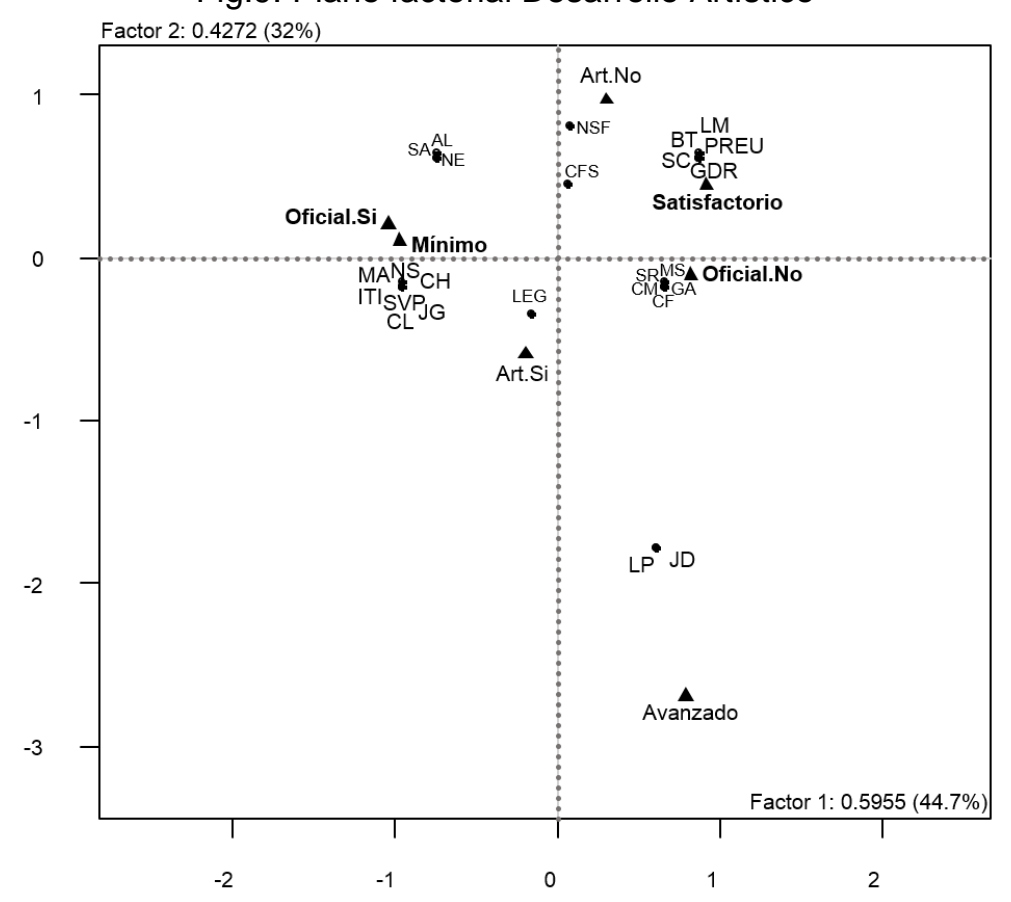

Fuente: Elaboración propia. 
Se representa en un 76.7\% las variables iniciales. La inercia es de 1.0227, distribuida entre los dos primeros valores propios en un $76.7 \%$ (Fig. 9). Se tiene que el primer eje opone las instituciones MA, NS, CH, JG, CL, SVP e ITI con BT, LM, PREU, GDR y SC, en donde el primer grupo resulta en calificación mínima a pesar de ofrecer actividades de desarrollo artístico, hecho opuesto sucede en el segundo grupo, se haya en rango satisfactorio sin ofrecer tal servicio. Cabe resaltar que las instituciones que se encuentran en avanzado (no oficiales) desarrollan programas artísticos.

Como punto final, se estudia el plano factorial general, el cual recoge toda la información considerada y usada en el presente escrito. Se especifican, entre todas las variables, aquellas que mayor calidad de representación poseen para el estudio con lo que se dará una aproximación a los factores más influyentes a los resultados académicos.

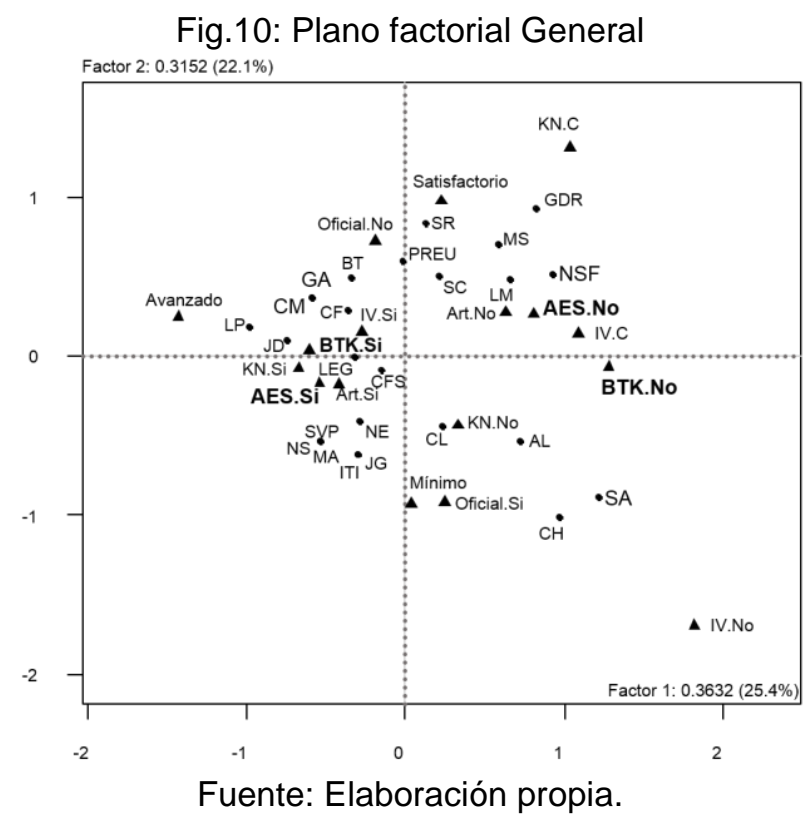

Con una inercia de 0.6784 dividida entre los dos primeros valores propios, representa todas las variables originales en un $47.5 \%$ (Fig. 10).

Es de notar que las variables de mayor contribución al análisis global de todos los individuos son la existencia o no de servicios de refuerzo estudiantil (S.R.E) y de bibliotecas. Hay mayor distribución de los individuos en cercanías de AES.NO y BTK.NO, es decir, en lejanías del centro de gravedad. Por el contrario, en los alrededores de BTK.SI y AES.SI se presenta una aglomeración de 
individuos.

\section{CONCLUSIÓN}

Tras haber investigado, organizado y analizado la información objeto de estudio de este trabajo, se llega a concluir que:

El impacto de las actividades complementarias y extraescolares a la calidad educativa en las instituciones oficiales con niveles de básica primaría en el municipio de Sincelejo es mínimo, muchas de ellas con distintas actividades de esta índole mantienen un nivel académico mínimo. La existencia de instalaciones científicas y deportivas, la implementación de un servicio de refuerzo estudiantil, las metodologías de recuperación y nivelación, las dotaciones bibliográficas y la realización de eventos artísticos y deportivos parecen no contribuir al mejoramiento de los índices académicos. Datos que verifican lo mencionado son por ejemplo que el $72.7 \%$ tienen al menos una instalación virtual, el 63.6\% cuenta con S.R.E y el $54.5 \%$ con bibliotecas, sin embargo, el $90.9 \%$ no supera las competencias mínimas.

Para el caso de las escuelas no oficiales por lo general niveles satisfactorio y avanzado están acompañados en un alto porcentaje de actividades complementarias y extraescolares. Cabe mencionar que las características y condiciones en las cuales son dadas tales actividades son muy similares a como se dan en las oficiales, los tiempos de servicio de las bibliotecas, las metodologías de tutorías externas, los lugares de refuerzo y las intensidades horarias para llevar a cabo diversas acciones pedagógicas son parecidas.

\section{AGRADECIMIENTOS}

Este trabajo ha sido realizado gracias al convenio entre la Universidad de Sucre y Colciencias en el cual se busca formar jóvenes investigadores e innovadores profesionales, mediante el otorgamiento de una beca pasantía en la modalidad tradicional. 


\section{REFERÊNCIAS}

Álvarez, j., m. Palomar, m. Vilches yb. Lainez, actividades y aprendizaje propuestos para tecnología. Http://www.eduinnova.es/ene 2010/actividadestec.pdf. Acceso: 7 de julio (2013).

Barragán, c. Y j. Ruiz, aplicación de las nuevas tecnologías a la educación infantil y primaria (2001), http://www.it.uc3m.es . Acceso: 17 de noviembre (2013).

Bolívar, a. Y moreno, j. M. Between transaction and transformation: the role of school principals as education leaders in spain, en journal of educational change. (2006).

Cantero, n. El papel integrador de la educación artística en educación primaria, revista innovación y experiencias educativas, no 39(2011).

Cervini, rubén, dari, nora, \& quiroz, silvia. Estructura familiar y rendimiento académico en países de américa latina: los datos del segundo estudio regional comparativo y explicativo. Revista mexicana de investigación educativa, 19(61), 569-597. (2014).

Cladellas, r., clariana, m., badia, m., gotzens, c. Actividades extraescolares y rendimiento académico en alumnos de primaria. Revista european journal of investigation in health, psychology and education, vol. 3, $n^{\circ}=2$, pp 87-97. (2013).

Clariana, r.b., wolfe, m.b. \& kim, k. The influence of narrative and expository lesson text structures on knowledge structures: alternate measures of knowledge structure. Education tech research dev62, 601-616 (2014). Https://doi.org/10.1007/s11423-014-9348-3

Corrales, a. El deporte como elemento educativo indispensable en el área de educación

física, http://emasf.webcindario.com/el_deporte_como_elemento_educativo_en_ef.pdf ; issn: 1989-8304, rev. Digital de educación física, 4,1-14 (2010).

Escudero, j.m el gobierno y la gestión de los centros y la calidad de la educación para todos. Congreso sobre "el centro educativo como núcleo de innovación y cambio". Santander. (2005)

Mijangos, j, la importancia de la educación motriz en el proceso de enseñanza de la lectoescritura en niños del nivel preprimario y de primero de primaria en escuelas oficiales del municipio de quetzaltenango, trabajo de investigación, universidad rafael landivar (2005).

Navas, I., maicas, g. S., \& germán, m. A. S. Predicción de las calificaciones de los estudiantes: la capacidad explicativa de la inteligencia general y de la motivación. Revista de psicología general y aplicada: revista de la federación española de asociaciones de psicología, 56(2), 225-237. (2003). 
Pardo, c. Y g. Cabarcas, métodos estadísticos multivariados en investigación social, simposio de estadística (2001).

Pardo, c.y p. Del campo,combinación de métodos factoriales y de análisis de conglomerados en $r$ : el paquete factoclass, revista colombiana de estadística 30 (2007).

Peacock, m. La biblioteca de la escuela primaria y sus servicios, http://unesdoc.unesco.org/images/0013/001316/131619so.pdf. Acceso: 23 de octubre (2013).

Pereira, f. "reflexiã"n sobre algunas caracterã sticas del espã ritu emprendedorcolombiano," economãa, gestiãn y desarrollo 003422, universidad javeriana - cali. (2003).

R development core team. R: a language and environment for statistical computing, $r$ foundation for statistical computing, vienna, austria. Isbn 3- 900051-07-0. Http://www.r-project.org (2015).

Raimundi, m., molina, m., giménez, m. \& minichielo, c. ¿qué es un desafío? Estudio cualitativo de su significado subjetivo en adolescentes de buenos aires. Revista latinoamericana de ciencias sociales, niñez y juventud, 12 (2), pp. 521-534. Doi: 10.11600/1692715x. 1221110414. (2014).

Rivière, m. Claves del programa de acompañamiento escolar, en portada, $\mathrm{n}^{\circ} 65$, 24-40 (2008).

Schmidt, j. Y padilla, b. Self-esteem and family challenge: an investigation of their effects on achievement. Journal of youth and adolescence, 32 (1), pp. 3746.doi:10.1023/a:1021080323230. (2003).

Thioulouse, j., a. Dufour y d.chessel,ade4: analysis of environmental data: exploratory and euclidean method multivariate data analysis and graphical display, lyon, france(2004). 